\title{
Analysis of Approximations and Aperture Distortion for 3D Migration of Bistatic Radar Data with the Two-Step Approach
}

\author{
Luigi Zanzi and Maurizio Lualdi \\ Dipartimento di Ingegneria Strutturale, Politecnico di Milano, Piazza Leonardo Da Vinci 32, 20133 Milano, Italy \\ Correspondence should be addressed to Luigi Zanzi, luigi.zanzi@polimi.it
}

Received 31 December 2009; Accepted 7 June 2010

Academic Editor: João Manuel R. S. Tavares

Copyright (๑) 2010 L. Zanzi and M. Lualdi. This is an open access article distributed under the Creative Commons Attribution License, which permits unrestricted use, distribution, and reproduction in any medium, provided the original work is properly cited.

\begin{abstract}
The two-step approach is a fast algorithm for 3D migration originally introduced to process zero-offset seismic data. Its application to monostatic GPR (Ground Penetrating Radar) data is straightforward. A direct extension of the algorithm for the application to bistatic radar data is possible provided that the TX-RX azimuth is constant. As for the zero-offset case, the two-step operator is exactly equivalent to the one-step $3 \mathrm{D}$ operator for a constant velocity medium and is an approximation of the one-step $3 \mathrm{D}$ operator for a medium where the velocity varies vertically. Two methods are explored for handling a heterogeneous medium; both are suitable for the application of the two-step approach, and they are compared in terms of accuracy of the final 3D operator. The aperture of the two-step operator is discussed, and a solution is proposed to optimize its shape. The analysis is of interest for any NDT application where the medium is expected to be heterogeneous, or where the antenna is not in direct contact with the medium (e.g., NDT of artworks, humanitarian demining, radar with air-launched antennas).
\end{abstract}

\section{Introduction}

In 1983, Gibson et al. [1] introduced the fast two-step migration technique for $3 \mathrm{D}$ poststack seismic data. In a companion paper, Jakubowicz and Levin [2] showed that in a constant velocity medium the method is equivalent to the classical one-step 3D migration. In their paper, Gibson et al. performed a detailed analysis of the differences between the two-step approach and the one-step approach when the velocity varies within the medium. They showed that in normal conditions these differences are negligible so that the method was suggested as a quite attractive solution for fast 3D migration of poststack seismic data. The extension of the two-step approach to prestack 3D migration is not straightforward although achievable as shown by Canning and Gardner [3]. They proposed a scheme composed of 3D $\mathrm{DMO}$, cross-line 2D PSI, inline 2 $\mathrm{D} \mathrm{DMO}^{-1}$, velocity analysis, and $2 \mathrm{D}$ inline depth migration. A variation of this scheme was proposed by Meinardus et al. [4]

Here, it is shown that under a very restrictive condition, that is, when the source-receiver azimuth is constant, the two-step approach can be directly extended to non-zerooffset data. In seismics, this would be the case of 3D marine data collected by a single ship, equipped with a single cable, and shooting along parallel lines. Of course this is not very interesting for the seismic community where 3D acquisitions are designed aiming at a balanced azimuth distribution to get a good picture of $3 \mathrm{D}$ structures. Instead, the result is interesting for GPR applications where 3D experiments are normally executed by maintaining a constant orientation of the antenna box, that is, a constant TX-RX azimuth. With the present hardware technology, this approach is what is needed by GPR users to achieve the goal of real-time visualization of 3D migrated volumes. Thus, the following sections discuss the non-zero-offset extension of the two-step method, the approximations resulting from the application to vertically variable velocity fields, and finally the distortion effects on the aperture of the migration operators. The quantitative results are derived assuming the usage of an ultra highfrequency radar with air-launched antennas. This type of hardware is normally preferred to ground-coupled antennas to speed up the NDT acquisitions on highways and bridges. It 


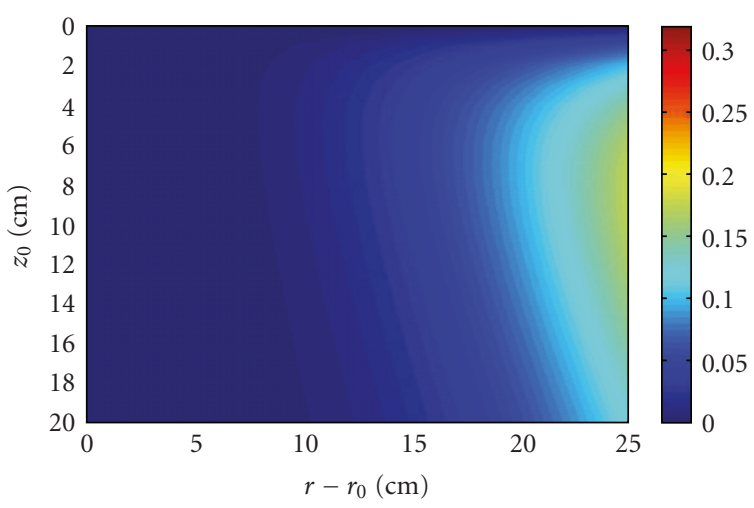

(a)

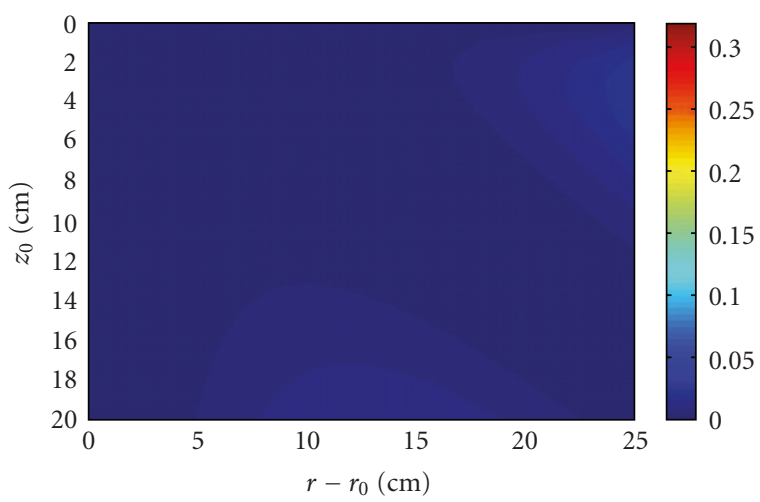

(b)

FIgURE 1: Travel time error [ns] for the rms approximation (a) and for the LLNL approximation (b) as a function of $z_{0}$ (target depth) and $r-r_{0}$ (lateral distance of the antenna from the vertical above the target). The system is monostatic. The antenna-medium distance is $10 \mathrm{~cm}$, and the radar wave velocity into the medium is $12 \mathrm{~cm} / \mathrm{ns}$.

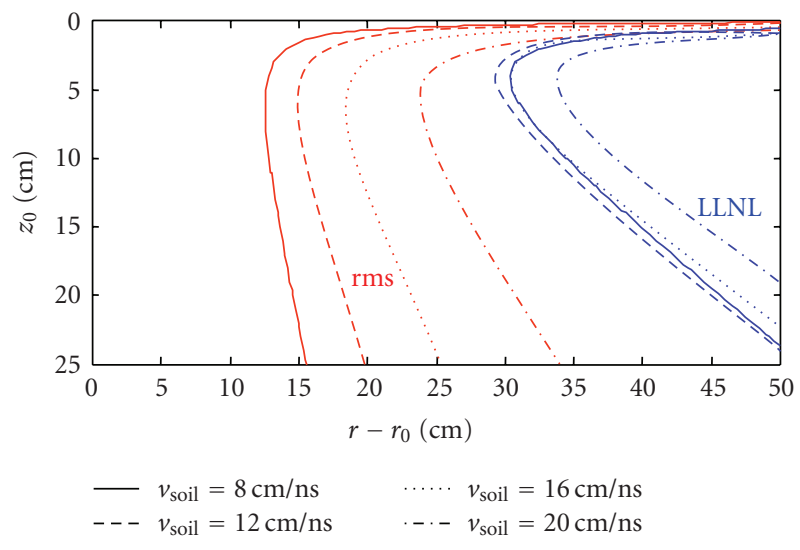

Figure 2: Migration aperture $\left(r-r_{0}\right)$ for the rms and the LLNL approximations as a function of $z_{0}$ (target depth). The antennamedium distance is $10 \mathrm{~cm}$, and the radar wave velocity into the medium is examined in the range from 8 to $20 \mathrm{~cm} / \mathrm{ns}$. The aperture is limited to the contributions affected by a phase error at the highest frequency $(6 \mathrm{GHz})$ lower than $\pi / 2$.

is also preferred for humanitarian demining to prevent mine activation and for diagnostic inspections on cultural heritage and artworks to prevent damages to delicate decorations, paintings, precious materials, and so forth. The air gap that separates the antenna from the medium generates a situation where the migration velocity field varies vertically even if the medium is homogeneous. Nevertheless, the discussion that follows is also of interest for radars with ground-coupled antennas when they are used to investigate a medium that is vertically heterogeneous. This is a frequent situation when a GPR is applied to NDT inspections of layered structures such as walls, floors, and pavements.

\section{The Two-Step Approach}

The two-step approach originally introduced by Gibson et al. [1] is exact in a constant velocity medium explored with

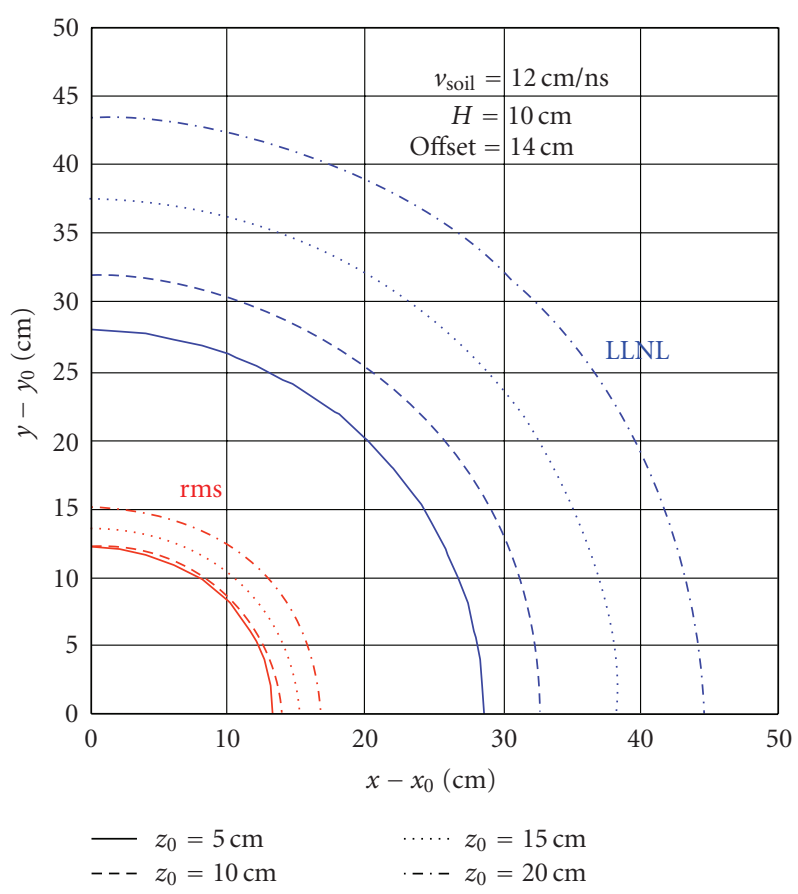

Figure 3: Migration aperture with the one-step approach for the rms and the LLNL approximations at four different target depths. The antenna-medium distance is $10 \mathrm{~cm}$; the radar wave velocity into the medium is $12 \mathrm{~cm} / \mathrm{ns}$; the TX-RX distance is $14 \mathrm{~cm}$ with the azimuth oriented in the $y$-direction. A small distortion of the circular shape is observed on the migration aperture as a result of the TX-RX separation.

a zero-offset experiment, for example, with a monostatic radar system. In this case, a scattering point located at $P_{0}\left(x_{0}, y_{0}, z_{0}\right)$ in the model space will produce a $3 \mathrm{D}$ diffraction surface in the data space given by

$$
t^{2}=T_{0}^{2}+\frac{4\left(x-x_{0}\right)^{2}}{v^{2}}+\frac{4\left(y-y_{0}\right)^{2}}{v^{2}}
$$




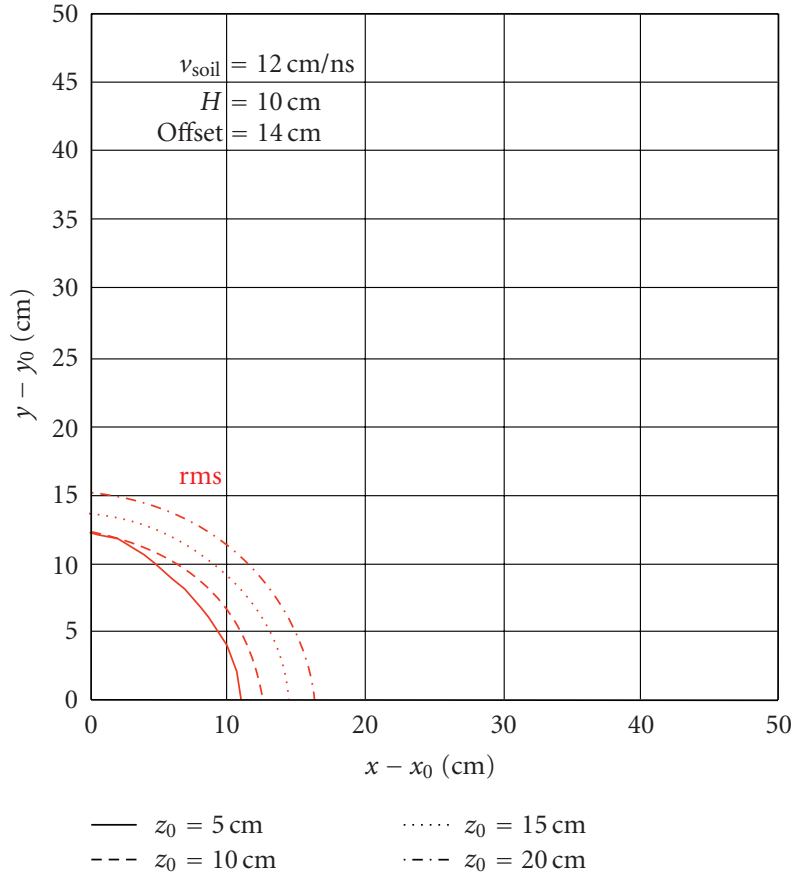

(a)

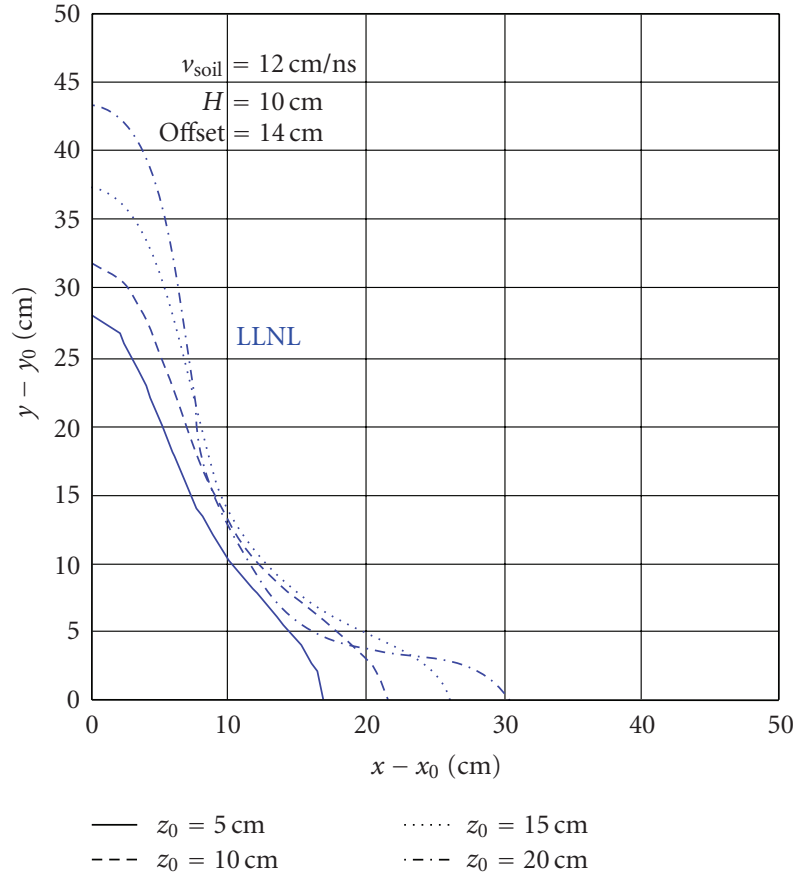

(b)

FIGURE 4: Migration aperture with the two-step approach for the rms (a) and the LLNL (b) approximations at four different target depths. The antenna-medium distance is $10 \mathrm{~cm}$; the radar wave velocity into the medium is $12 \mathrm{~cm} / \mathrm{ns}$; the TX-RX distance is $14 \mathrm{~cm}$ with the azimuth oriented in the $y$-direction.

where $T_{0}=2 z_{0} / v$. The diffraction surface is a hyperboloid, and its intersection with a vertical plane, for example, with a plane parallel to the $y$-axis, is a hyperbola given by

$$
t^{2}=t_{0}^{2}+\frac{4\left(y-y_{0}\right)^{2}}{v^{2}}
$$

where

$$
t_{0}^{2}=T_{0}^{2}+\frac{4\left(x-x_{0}\right)^{2}}{v^{2}}
$$

The two-step approach consists of performing a 2D migration in the $y$-direction according to (2) followed by a $2 \mathrm{D}$ migration in the $x$ direction according to (3). Note that in the monostatic case any summation order is valid.

Let us consider now a bistatic system where the TX-RX separation is $2 d$, and let us assume that the $3 \mathrm{D}$ experiment is executed by keeping a constant TX-RX azimuth. If we rotate the coordinate system in such a way that the $y$-direction is the azimuth direction, the $3 \mathrm{D}$ diffraction surface will be now given by

$$
\begin{aligned}
t= & \sqrt{\left(\frac{T_{0}}{2}\right)^{2}+\frac{\left(x-x_{0}\right)^{2}}{v^{2}}+\frac{\left(y-y_{0}-d\right)^{2}}{v^{2}}} \\
& +\sqrt{\left(\frac{T_{0}}{2}\right)^{2}+\frac{\left(x-x_{0}\right)^{2}}{v^{2}}+\frac{\left(y-y_{0}+d\right)^{2}}{v^{2}}} .
\end{aligned}
$$

The diffraction surface is no more a hyperboloid, and its intersection with a vertical plane parallel to the $y$-axis is

$$
t=\sqrt{\left(\frac{t_{0}}{2}\right)^{2}+\frac{\left(y-y_{0}-d\right)^{2}}{v^{2}}}+\sqrt{\left(\frac{t_{0}}{2}\right)^{2}+\frac{\left(y-y_{0}+d\right)^{2}}{v^{2}}},
$$

where

$$
\left(\frac{t_{0}}{2}\right)^{2}=\left(\frac{T_{0}}{2}\right)^{2}+\frac{\left(x-x_{0}\right)^{2}}{v^{2}} .
$$

The extension of the two-step approach consists of performing a $2 \mathrm{D}$ non-zero-offset migration in the $y$-direction according to (5) followed by a $2 \mathrm{D}$ zero-offset migration in the $x$ direction according to (6). Note that in the bistatic case the summation order is relevant, that is, the first step must be in the azimuth direction. The conclusion is that the extension of the two-step approach to a homogeneous medium investigated with a bistatic radar is possible, and the algorithm is totally equivalent to an exact one-step 3D migration.

\section{Approximations for the Vertically Heterogeneous Medium}

The standard method to migrate the diffractions observed in a medium where the velocity varies vertically consists of using the rms velocity function to extend the use of the equations derived for the constant velocity medium. Another 


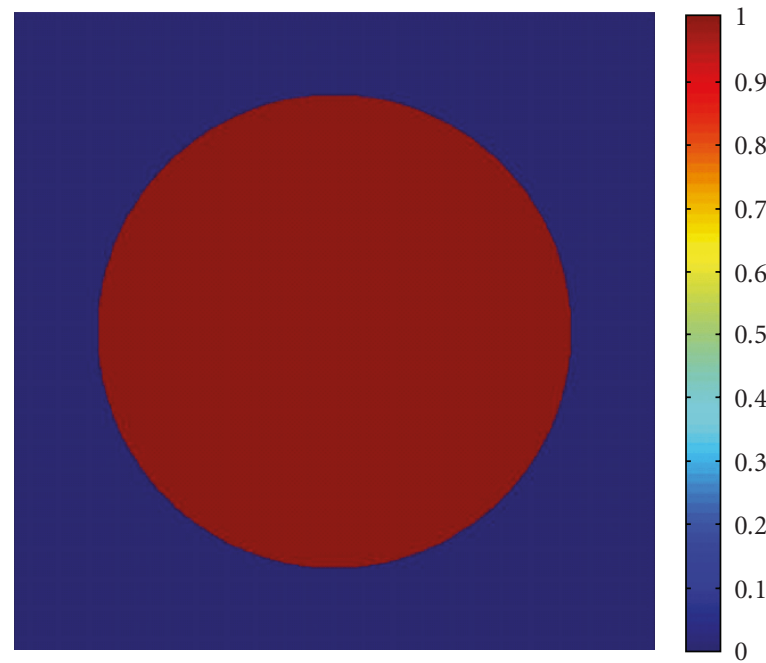

(a)

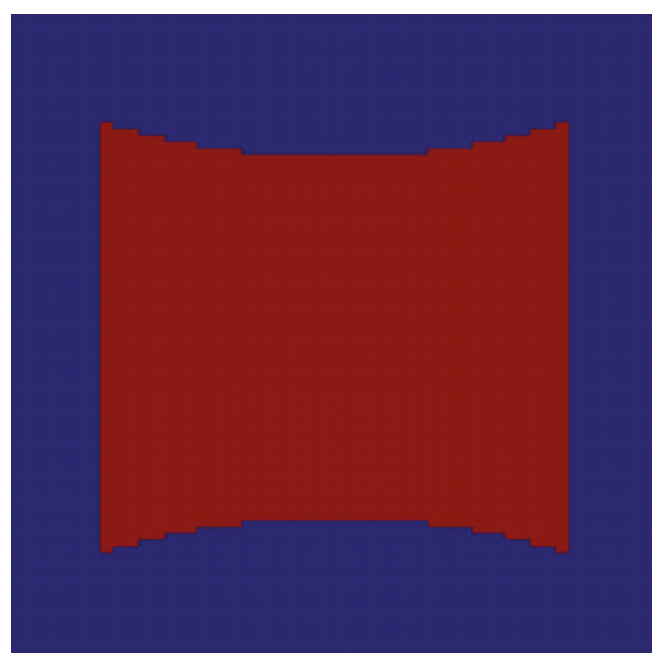

(c)

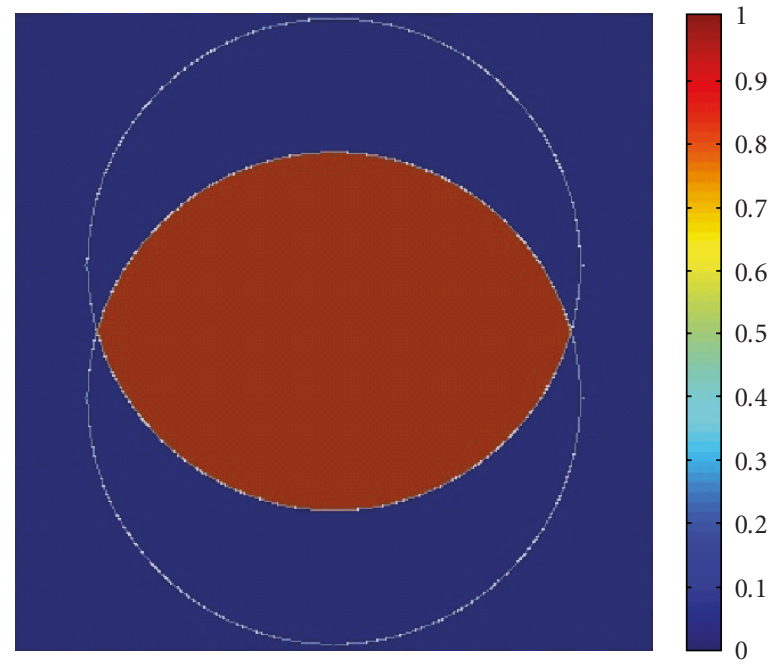

(b)
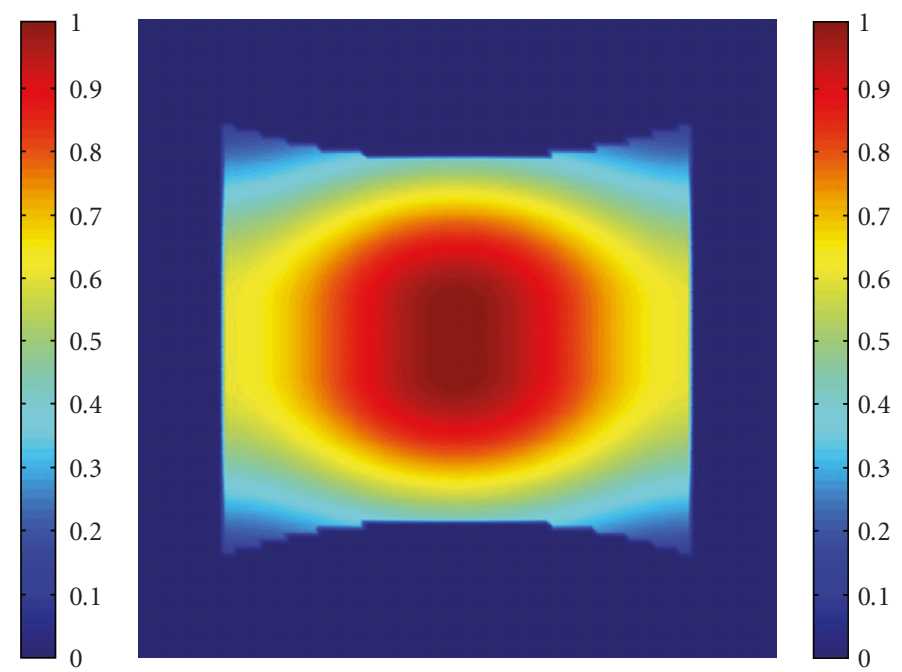

(d)

FIGURE 5: Different forms that can be used to shape the migration operator aperture in order to resemble the real radar footprint; (a) and (b) are applicable to the one-step 3D operator to simulate a monostatic and a bistatic footprint, respectively; (c) is the aperture of the twostep operator obtained with a trivial 2D aperture limitation applied to both the $x$ and $y$-direction steps; (d) is the aperture of the two-step operator that can be obtained with a smooth weighting approach to resemble a shape similar to (b).

approach was successfully experimented at the Lawrence Livermore National Laboratories by Johansson and Mast [5]. This approach is applicable when the radar measurements are performed with air-launched horn antennas as those often used for nondestructive testing of highways and bridges, for humanitarian demining, for diagnostic investigations on art-works, and so forth. The air gap that separates the antenna from the medium generates a situation where the migration velocity field varies vertically even if the medium is homogeneous. Johansson and Mast proposed a method based on an approximate estimation of the inflection point, that is, the point on the medium surface where the antenna-target raypath is bent according to Snell's law. Both methods preserve the property discussed above, that is, the possibility to split the $3 \mathrm{D}$ migration operation into a sequence of two bidimensional migrations provided that a proper order is followed when the system is bistatic. Let us examine both approximations, shortly indicated in the following as rms and LLNL solutions, and let us perform a kinematical analysis of the expected errors with respect to the exact 3D migration. We will see that the final errors are the combination of the errors induced by the approximation adopted to estimate the diffraction surface plus the additional errors induced by the application of the two-step approach. Thus, let us discuss first the errors for the monostatic case and the bistatic case when the migration is performed in one step, and then let us consider the additional errors introduced by the two-step approach.

Starting with the monostatic system, Figure 1(a) shows an example of the travel time error, that is, the difference 


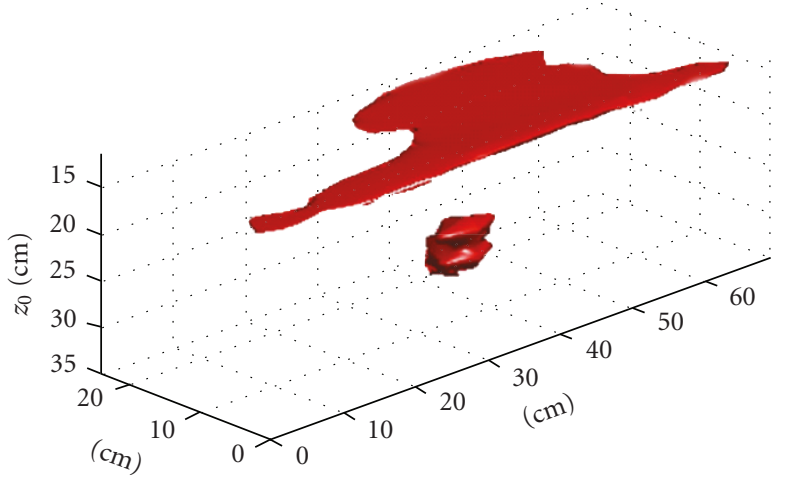

FIGURE 6: 3D reconstruction of a plastic antipersonnel mine obtained by applying the two-step migration algorithm to real laboratory data. The mine is buried $10 \mathrm{~cm}$ below the surface of a sand box. The frequency range is from 2 to $6 \mathrm{GHz}$; the TX-RX distance is $14 \mathrm{~cm}$; the antenna is moved $13 \mathrm{~cm}$ above the soil. The $3 \mathrm{D}$ reconstruction is a $3 \mathrm{D}$ contour of a selected amplitude of the migrated signal that emphasizes the energy reflected by the sand surface and the energy scattered by the mine.

between the exact and the approximated diffraction surface, obtained with the rms approach. In the same way we can explore the LLNL approximation and, as claimed by Johansson and Mast [5], a lower error level is to be expected (Figure 1(b)). As a result, we also expect that a larger aperture of the migration operator can be selected for the LLNL method. For a quantitative comparison let us conventionally limit the migration aperture to the circular area where the phase errors of the highest frequency contributions do not exceed $\pi / 2$. The aperture comparison is shown in Figure 2, assuming, as an example, that the highest frequency is $6 \mathrm{GHz}$.

Let us consider now a bistatic system. The travel time error is still expected to be a function of the target depth and of the lateral displacement of the antenna box from the vertical above the target, but a further parameter will influence the error: the azimuth direction. As a result, the limit of the migration aperture varies with the direction from which the contributions come. This is shown in Figure 3 where the migration aperture for the one-step approach is plotted on horizontal planes at four different target depths.

Finally, let us extend the error analysis to the two-step approach. With respect to the one-step approach, we have to include a further error, that is, the difference between the $3 \mathrm{D}$ approximation of the diffraction surface and the actual diffraction surface over which the contributions are taken when the two-step approach is applied. For the rms approximation, the difference comes from the velocities that are applied to perform step 1 with (5) and step 2 with (6): in principle, both of these velocities should be equal to the rms velocity observed at the zero-offset time $T_{0}$, whereas in practice the velocity applied for the first step is the rms velocity observed at a higher zero-offset time given by $t_{0}$. In other words, the problem is due to the fact that when the velocity varies vertically, for a given $t_{0}$, the intersection of the one-step 3D diffraction surface with a vertical plane parallel to the $y$-axis, (5) is not only a function of $t_{0}$ but also depends on the zero-offset time $T_{0}$ (see (6)). A similar comment is applicable to the LLNL approach. Again the problem comes from the fact that the first step should collapse in $t_{0}$ contributions that belong to different intersection curves depending on the final $T_{0}$ where the diffraction is going to be focused.

As an example, Figure 4 shows how the migration aperture of Figure 3 is further reduced when the two-step approach is applied. Again we see that the LLNL approximation is more accurate than the rms approximation but the gap is now less remarkable than in Figure 3, meaning that the rms approximation is particularly robust with respect to the degradation introduced by the two-step approach.

Finally, let us remind the reader that the migration aperture that we are discussing here is the aperture that we would like to select to perform a constructive interference of the summed contributions. As we are going to see in the next section, the actual shape of the migration aperture that we can obtain with the two-step approach might be very different. Besides, we want to stress the point that the aperture discussed in this section has nothing to do with the real footprint of the radar system, that is, with the area actually illuminated by the antenna that depends on many other factors related with the antenna distance from the medium, the medium absorption, the medium permittivity, and so forth. Nevertheless, the results of the analysis are encouraging because the real footprint measured on experimental data with physical parameters similar to those assumed in the above examples is seldom wider than the conservative aperture of the two-step operator suggested by Figure 4 to prevent destructive interference of unfocused data.

\section{Design of the Operator Aperture}

When 3D migration is performed in one step, the aperture of the operator can be designed according to any desired shape, for example, as a circle or an ellipse (Figure 5(a)) to resemble a monostatic footprint, or as an intersection of circles or ellipses to resemble a bistatic footprint (Figure 5(b)). Instead, the two-step approach is strongly limited regarding this aspect. Gibson et al. [1] pointed out that the operator aperture cannot be circular or elliptical, rather, a rectangular shape would result if a constant limit is applied to both the $x$ and the $y$ migration steps. Furthermore, if this limit is time variant in following the expected footprint increase with depth, a characteristic distortion of the rectangular shape is expected (Figure 5(c)). A low cost solution that we propose in order to return to pseudo-elliptical apertures consists of applying a smooth weighting function rather than an on-off function to select the data that will contribute to focusing a point in the migrated space. For example, if the $W(x, y)$ weighting function is obtained as $W(x, y)=W_{x}(x) * W_{y}(y)$, so that it is suited for the two-step approach, and both $W_{x}$ and $W_{y}$ are designed as smooth functions, for example, Hanning functions, the $3 \mathrm{D}$ smooth aperture of the two-step operator will appear as in Figure 5(d). 


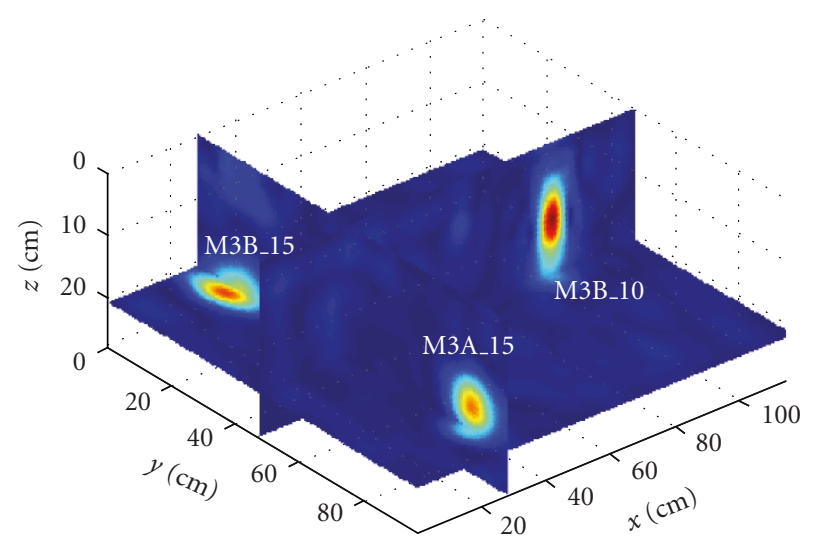

(a)

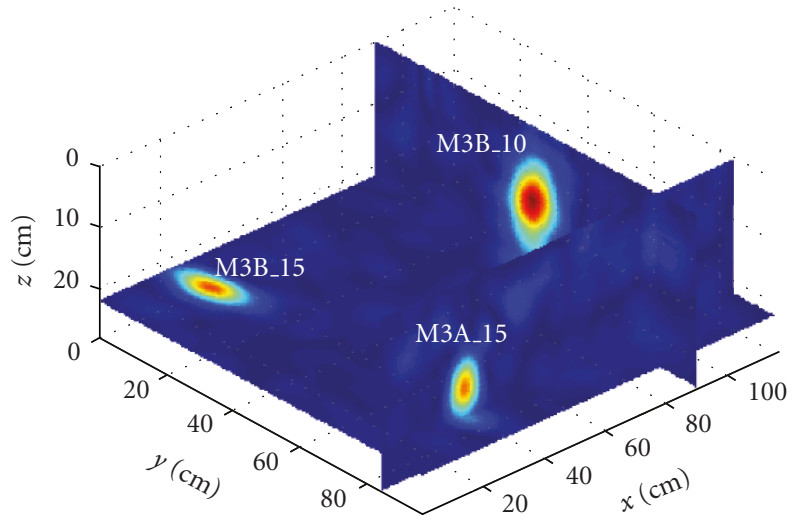

(c)

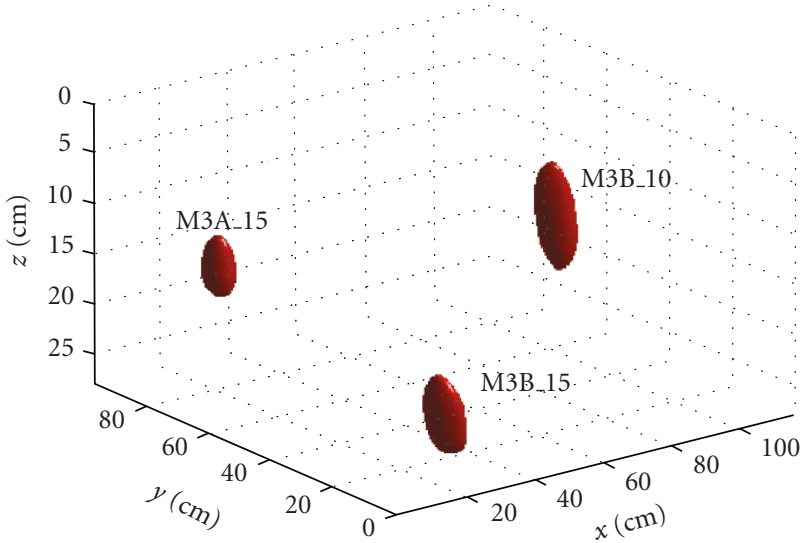

(b)

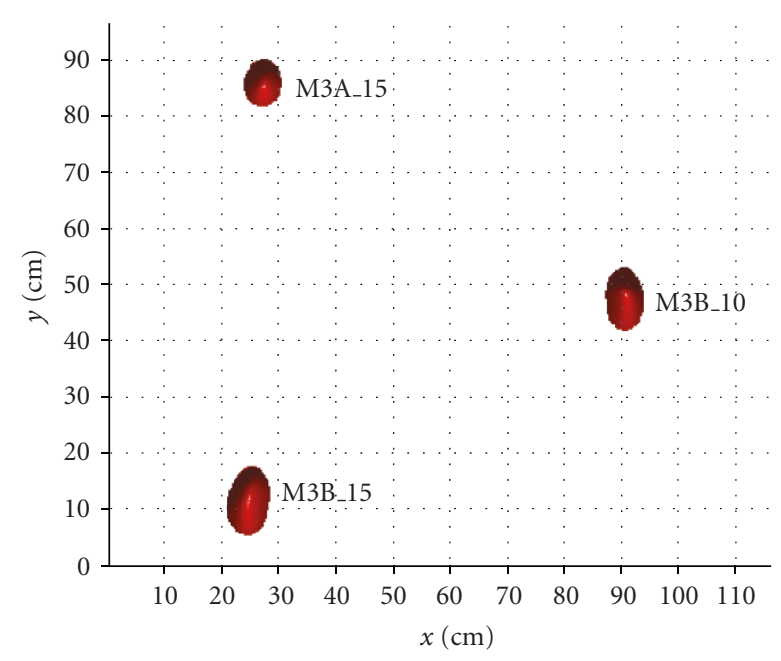

(d)

FIgURE 7: 3D representations through cross-sections (a, c) and through iso-amplitude plots (b, d) of the focused data over a sector of the clay box of the JRC mine field. The sector explored with a $1 \mathrm{GHz}$ antenna contains three mines buried at $10 \mathrm{~cm}$ and $15 \mathrm{~cm}$. M3B indicates mines with low metal content, while M3A indicates mines with high metal content.

\section{Application Examples}

A few examples are shortly presented to illustrate situations where the vertical heterogeneity of the medium is successfully handled by using the two-step migration approach with the rms velocity approximation to focus the data collected with high-frequency bistatic radar systems.

Figure 6 presents the 3D image of a nonmetal dummy mine (diameter $11 \mathrm{~cm}$, thickness $6 \mathrm{~cm}$ ) buried $10 \mathrm{~cm}$ in a sand box. The target was explored with a stepped frequency radar prototyped by RST. The area was manually scanned by executing parallel profiles with a TX-RX aperture of $14 \mathrm{~cm}$ and keeping the air-launched antennas at an approximate height of $13 \mathrm{~cm}$ above the sand surface. The final scanning grid was approximately $0.8 \mathrm{~cm}$ in the profile direction and $3 \mathrm{~cm}$ in the orthogonal direction. The frequency range was $2-6 \mathrm{GHz}$ with a frequency step of $16 \mathrm{MHz}$. Despite of the very low permittivity contrast between the sand and the mine material, the weak signal scattered by the target was successfully collapsed by the migration operator producing a final focused image where the mine energy is sufficiently higher than the noise.

The second example is taken from a test performed at the landmine field prepared at the Joint Research Center (JRC) in Ispra (Italy). The test site was designed to evaluate the performances of any new equipment proposed for humanitarian demining. The field consists of seven boxes with different soils; every box is $6 \mathrm{~m}$ long and $5.7 \mathrm{~m}$ wide and is prepared by reproducing exactly the same scheme of buried mines or objects. Here we collected some 3D data with a $1 \mathrm{GHz}$ commercial antenna controlled by a pulse radar unit from Mala. The final goal was to explore the effect of a $2 \mathrm{~cm}$ layer of ballistic material used to cover a small sector of the investigation area (e.g., $1 \times 1 \mathrm{~m}$ ) in order to protect the operator and the radar antenna in case of a landmine activation. The GPR profiles were manually performed by sliding the antenna over the ballistic protection layer. Of course, the ballistic material was chosen among materials with good dielectric properties and with low density so that the radar signal is not absorbed, and the weight of the 


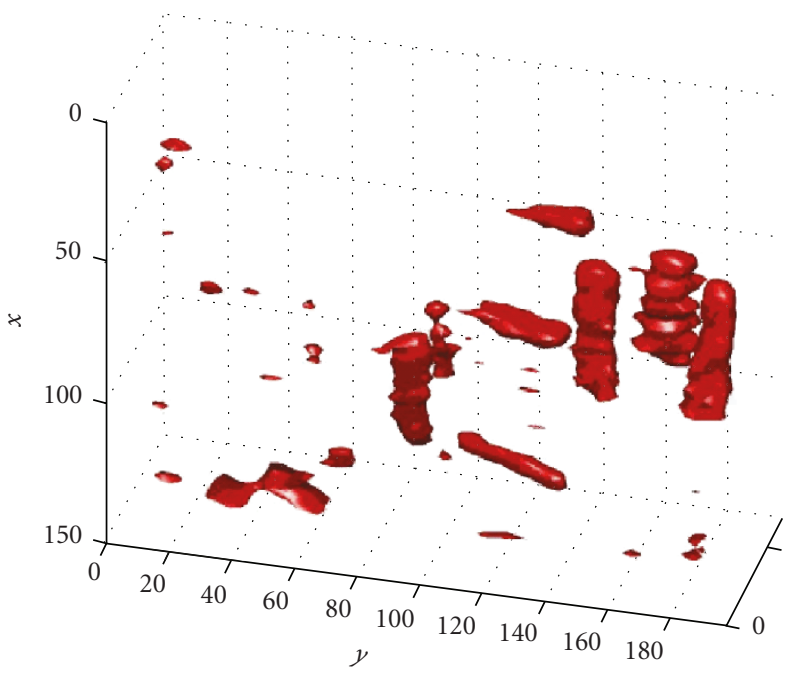

(a)

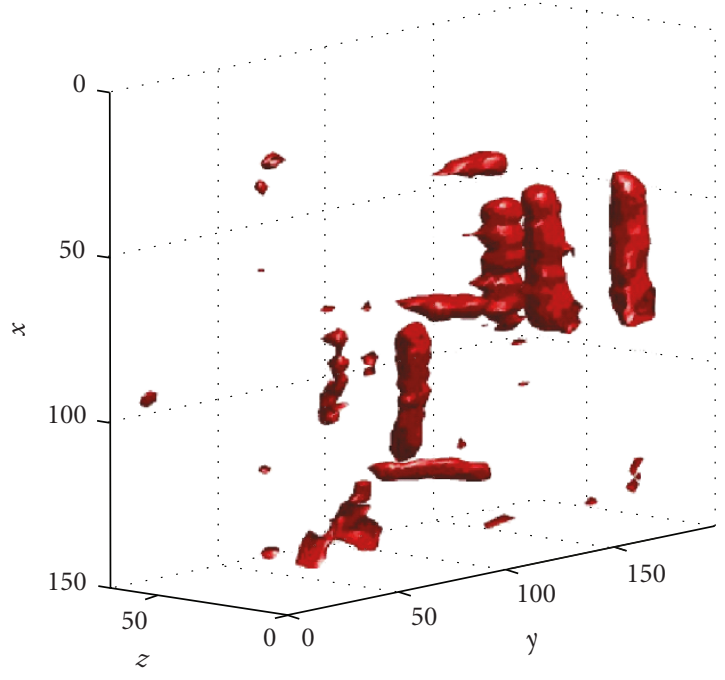

(b)

Figure 8: 3D reconstruction of the joints that were used to connect the marble fragments when the monument was rebuilt in the 20th century. Data collected by sliding a $1 \mathrm{GHz}$ antenna over a cardboard in order to preserve the integrity of the delicate carvings that decorate the explored wall. The focused data are displayed by showing from two different perspectives the 3D contour of an iso-amplitude surface. Dimensions are indicated in $\mathrm{cm}$.

armored pad plus the weight of the antenna are not expected to trigger a landmine. The $3 \mathrm{D}$ processed data are shown in Figure 7 using two different representation methods. The quality of the final results is quite good considering that these data were collected on the unfavorable clay soil box. The result was also compared with data collected without the protection pad validating the expectation that the ballistic material does not introduce any significant degradation. This is also a demonstration that the two-step migration operator was properly dealing with the heterogeneity of the medium consisting of two different materials: the ballistic layer and the clay soil.

The third example is a 3D survey of a wall of a marble monument built in Rome in $13 \mathrm{BC}$. The fragments of the monument were found during archeological excavations, and the monument was rebuilt in the third decade of the 20th century. Unfortunately, some details about the reconstruction are missing. The data presented in Figure 8 were collected with a GSSI pulse radar equipment by scanning the wall with a $1 \mathrm{GHz}$ antenna. A cardboard was interposed between the antenna and the wall in order to create a flat surface and preserve the delicate carvings that decorate the wall. As a result, the antenna is partially detached by the wall. Again, this situation creates a material heterogeneity that can be roughly assimilated to a two-layer structure where the first layer is of a few centimeters and is mainly consisting of voids, and thus is very fast for the radar signal, while the second layer consists of marble. The focused data reveal the existence of few metallic bars that were used to connect the marble fragments.

The last example is taken from an investigation performed on a historical Palace in Venice in order to map the position and length of hidden iron connection devices that have been used until the nineteenth century in Venetian building to link the wooden floors to the masonry external façades. These metal joints were called "fiube" and were either nailed to the floor planks or nailed to the timber beams supporting the wooden floor [6]. The heads of these metal elements are sometimes visible on the external façade; other times they are hidden inside the stone masonry structure or are hidden by the plaster or by ornamental elements decorating the façade. This was the case of the investigated Palace where a high-frequency $(2 \mathrm{GHz})$ GPR system was used to locate these elements by surveying the floors of the Palace especially close to the external walls and in the corners of the building. Figure 9 shows an example of a $3 \mathrm{D}$ radar image after the application of the 3D migration algorithm. The floor in this room consists of a sort of ceramic pavement deposited over a timber floor consisting of planks supported by beams. As a result, the radar signal diffracted by the fiube travels in a heterogeneous material consisting of ceramics, timber, and void. The image presented in Figure 9 is a depth slice taken at $12.5 \mathrm{~cm}$ below the floor surface. At this depth the radar survey was intersecting two fiube with different headings, being the survey close to a building corner. The radar profiles were run in the direction perpendicular to the shorter fiuba. Thus, the profiles were intersecting the longer fiuba at an angle of about $45^{\circ}$. The well-focused images obtained for both metal elements, regardless of their orientation and regardless of the heterogeneity of the material, demonstrate the effectiveness of the 3D migration algorithm based on the two-step approach.

\section{Conclusions}

A direct extension of the two-step approach for fast 3D migration of bistatic GPR data is possible provided that the 

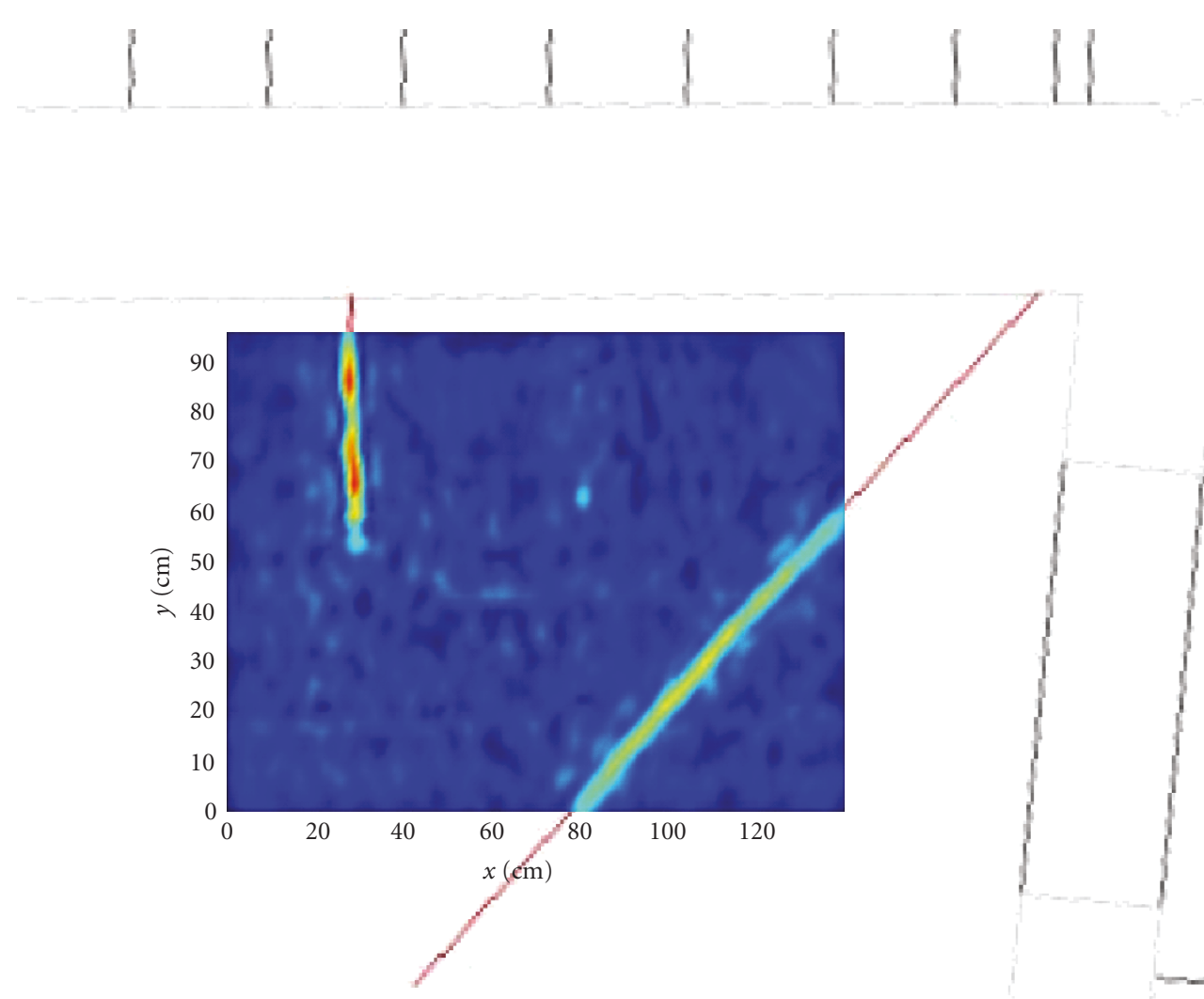

FIGURE 9: Depth slice at $12.5 \mathrm{~cm}$ below the floor surface extracted from a 3D radar survey performed with a 2-GHz antenna in proximity of a building corner of a historical palace in Venice. Data were processed with the two-step 3D migration algorithm. Two fiube (one coming from the building corner) appear in the radar image.

source-receiver azimuth is constant. As for the zero-offset case, the two-step operator is exactly equivalent to the onestep 3D operator for a constant velocity medium and is an approximation of the one-step 3D operator for a medium where the velocity varies vertically.

Two methods have been considered (rms and LLNL) for migrating data collected with air-launched antennas where the air gap that separates the antennas from the medium generates a situation that requires a vertically variable migration velocity even if the medium is homogeneous. Both methods are suitable for the application of the two-step approach, and they have been compared in terms of accuracy of the final 3D operator. The result of the analysis is that both the rms and the LLNL methods can be applied with the two-step approach producing a negligible degradation of the migration accuracy. A solution has been also proposed for an optimal shaping of the two-step operator aperture.

The impact of the two-step algorithm on the CPU cost of the $3 \mathrm{D}$ migration is quite interesting, as $3 \mathrm{D}$ images such as the mine reconstruction of Figure 6 can be produced in a few seconds, that is, in real time, with a standard personal computer. The advantage is of great interest if we consider that currently the GPR suppliers are producing multichannel GPR equipment with more and more antennas mounted in a cart to increase the productivity. These systems generate huge amounts of data that cannot be migrated in real time by a single computer unless a very effective algorithm is used.

Finally, the rms method and the accuracy discussion are also of interest when radars with ground-coupled antennas are used to investigate a medium that is vertically heterogeneous. This is a frequent situation when the GPR is applied to NDT inspections of layered structures such as walls, floors, and pavements.

\section{Acknowledgments}

The authors are grateful to RST GmbH that developed the stepped frequency radar prototype for humanitarian demining, to the Joint Research Center in Ispra that gave free access to the mine test field, to Dr. G. Lenzi of ISMES S.p.A. who performed the acquisitions on the marble monument in Rome, and to IDS S.p.A. that supplied the $2 \mathrm{GHz}$ system for the experiments in the Venetian Palace. 


\section{References}

[1] B. Gibson, K. Larner, and S. Levin, "Efficient 3-D migration in two steps," Geophysical Prospecting, vol. 31, no. 1, pp. 1-33, 1983.

[2] H. Jakubowicz and S. Levin, "A simple exact method of 3-D migration-theory," Geophysical Prospecting, vol. 31, no. 1, pp. 34-56, 1983.

[3] A. Canning and G. H. F. Gardner, "A two-pass approximation to 3-D prestack migration,” Geophysics, vol. 61, no. 2, pp. 409-421, 1996.

[4] H. Meinardus, C. Nieto, A. Chaveste, and J. Castañeda, "Efficient, target-oriented 3-D prestack depth migration in two steps," Leading Edge, vol. 19, no. 2, pp. 138-144, 2000.

[5] E. M. Johansson and J. E. Mast, "Three-dimensional groundpenetrating radar imaging using synthetic aperture timedomain focusing," in Advanced Microwave and Millimeter-Wave Detectors, vol. 2275 of Proceedings of SPIE, pp. 205-214, July 1994.

[6] G. Mirabella-Roberti, L. Zanzi, and F. Trovo', "Detecting hidden ties in historic venetian palace by means of GPR," in Proceedings of International RILEM Conference on Site Assessment of Concrete, Masonry and Timber Structures (SACoMaTiS '08), pp. 965-974, Varenna, Italy, September 2008. 